\title{
Aetiological agents of surgical site infection in a specialist hospital in Kano, north-western Nigeria
}

\author{
EMMANUEL O. NWANKWO ${ }^{1 *}$, CHARLES K. MOFOLORUNSHO ${ }^{1}$ and AZEEZ O. AKANDE ${ }^{2}$ \\ ${ }^{1}$ Department of Microbiology, Faculty of Natural Sciences, Kogi State University, Anyigba, Kogi State, Nigeria \\ ${ }^{2}$ Department of Microbiology and Parasitology, Faculty of Medicine, Bayero University, Kano, Nigeria
}

\begin{abstract}
Despite the advances made in asepsis, antimicrobial drugs, sterilization and operative techniques, surgical site infections (SSI) continue to be a major problem in all branches of surgery in the hospitals. The objective of this study was to establish the incidence of SSI, the type and frequency of various pathogens and their antibiotic susceptibility pattern in Murtala Mohammed Specialist Hospital (MMSH), Kano, Nigeria. A total of 2,920 consecutive patients who underwent different surgical procedures between January 2009 and December 2010 at Murtala Mohammed Specialist Hospital were enrolled in this study. Samples of pus were collected from infected cases and screened for bacterial and fungal pathogens by standard microbiological methods. Antibiotic susceptibility tests were done by disc diffusion technique. A total 878 patients were found to be clinically infected and 919 isolates were obtained in all. This gave an incidence of $30.1 \%$ infection rate. While $783(89.2 \%)$ cultures yielded single organism, $77(9.8 \%)$ yielded mixed growth and $18(8.1 \%)$ cultures yielded no growth. The most frequently isolated organism was E. coli (25.5\%) followed by Staphylococcus aureus 20.6\%. The incidence of Methicillin resistant Staphylococcus aureus was $35.7 \%$. Ceftriaxone, ceftazidime and ofloxacin showed good results against most isolates while ampicillin and cotrimoxazole which are commonly used drugs were ineffective. An understanding of the various types of pathogen involved in SSI and their antibiotic susceptibility pattern will reduce indiscriminate prescription of antibiotics and help in infection control.
\end{abstract}

Keywords: aetiology, surgical site, infection, antibiotics, susceptibility, Nigeria

\section{Introduction}

Despite the advances made in asepsis, antimicrobial drugs, sterilization and operative techniques, surgical site infections (SSI) continue to be a major problem in all branches of surgery in the hospitals (Linani et al., 2005). SSIs are responsible for the increasing costs and the morbidity and mortality which are related to surgical infections (Anvikar et al., 1999). The organisms which cause surgical infections vary from time to time and from place to place (Kumar et al., 1985). The pathogens that infect surgical wounds can be part of the patient's normal flora or they may be acquired from the hospital environment (Angue \& Olila, 1999).

In a study on occurrence of Pseudomonas aeruginosa in postoperative wound infection in Lagos, it was found that Pseudomonas aeruginosa was the most frequently isolated followed by Staphylococcus aureus (Oguntubeju \& Nwobu, 2004). The least isolated organism was Streptococcus Pyogenes and Enterococcus faecalis. Ejikeme (2004) also in a study of the bacteriology of postoperative wound infection in the surgical wards of a federal medical Centre, in Umuahia, South-Eastern Nigeria, found Pseudomonas aeruginosa as the most frequently isolated organism followed by Proteus spp. The least isolated organism was also Streptococcus pyogenes. Some other authors (Jan et al., 2010; Shah et al., 2010) reported E. coli as the most frequently isolated bacteria in their study

Collated data on the incidence of wound infections probably underestimate the incidence because some wound infections occur when the patient is discharged, and these infections may be treated in the community without hospital notification (Waqar et al., 2010). However, the incidence of infection varies from surgeon to surgeon, from hospital to hospital, from one surgical procedure to another and most importantly from one patient to another (Nichols, 2001).

*Correspondence: Emmanuel O. Nwankwo; Email: emmaonwubiko@yahoo.com 
The objective of this study was to determine the incidence and various types of pathogens involved in SSI in Murtala Mohammed Specialist hospital, Kano, Nigeria including their antibiotic susceptibility pattern which will be of clinical relevance in Kano City, there is dearth of current and relevant information on this subject

\section{Materials and Methods}

\section{Study area}

Murtala Mohammed Specialist hospital (MMSH) is a tertiary health care facility in the city of Kano North-western Nigeria with about 1000 beds. It provides a high level Medicare to a large population of people in a highly populated state. The study was carried out in male and female surgical wards, maternity, gynaecology and paediatric surgical wards where both emergency and elective procedures were performed.

\section{Study participants}

This prospective cross sectional study was carried out between January 2009 and December 2010. Patients who underwent various surgical procedures available in the hospital in the wards mentioned above were included in the surgery after informed consent. Orthopaedic surgical patients including those with implants were not included. Also infants and children between 0 10 years were also not included.

\section{Sampling and Sample size}

Samples of pus were carefully collected from clinically confirmed infected cases after cleaning the surrounding areas in eight hundred and seventy eight (878) patients out of a total number of 2920 patients who actually underwent surgery. A surgical site infection (SSI) case was identified using Centre for Disease Control (CDC) definition which states that infection would be regarded as an SSI if it occurs within 30 days of procedure and has at least one of the following purulent drainage from the wound, pain or tenderness, localized swelling, redness, malodour and fever. Patients that underwent procedure with implants were not enrolled in the study (Emori \& Gaynes, 1993).

\section{Laboratory sample and test}

A total number of 2,920 consecutive patients who underwent different surgical procedures between January 2009 and December 2010 at Murtala Mohammed Specialist Hospital (MMSH) were enrolled in this study. Samples of pus were collected from infected cases and screened for bacterial and fungal pathogens by standard microbiological methods. Antibiotic susceptibility tests were done by disc diffusion technique.

Eight hundred and seventy eight consecutive swab samples from post-operative infections were aseptically collected from patients in the male and female surgical ward, obstetrics and gynaecology including paediatric ward. Sterile swab sticks used were manufactured by Evepon Nigeria Limited and all the samples were processed at the Microbiology laboratory in $\mathrm{MMSH}$. The samples were inoculated on MacConkey, manitol salt, Blood and saboraud's dextrose agar for bacterial and fungal screening. Aerobic incubation was done at $37^{\circ} \mathrm{C}$ for 18-24hrs. Culture plates for fungal isolates were incubated at room temperature for upwards of $72 \mathrm{hrs}$. Isolates were identified by standard microbiological methods including gram stain, motility, oxidase, indole tests and other biochemical tests according to Cheesebrough (1993). Enterotube technique - Microbiology systems (BD BBL Enterotube II, UK) was used to identify isolates to species level. The control strains used were Staphylococcus aureus ATCC 25923, Escherichia coli ATCC 25922 and Pseudomonas aeruginosa ATCC 27853. 


\section{Sensitivity tests}

Sensitivity tests were carried out by the disc diffusion methods of Kirby-Bauer and compared with standard isolates (Bauer et al., 1996). The swab stick sample for anaerobes were inoculated immediately after aseptic collection onto neomycin sulphate blood agar and cooked meat medium for anaerobic bacteria and incubated at $37^{\circ} \mathrm{C}$ for $48-72 \mathrm{hrs}$. The cooked meat medium was sub-cultured on neomycin sulphate blood agar. The culture plates for anaerobes were put into an anaerobic jar (BBL gar pack system, Oxoid) and procedures carried out according to the

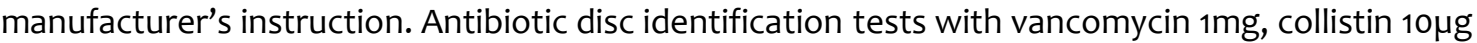
and vancomycin $5 \mu \mathrm{g}$ (Oxoid) were used for the preliminary grouping of anaerobes. Bile disc was used for Bacteroides fragilis while penicillin and novobiocin discs (oxoid) were equally used for anaerobic cocci according to the methods of Baron et al. (1994).

The susceptibility of consecutive isolates of S. aureus to oxacillin was determined on Mueller-Hinton agar supplemented with $2 \% \mathrm{NaCl}$. Plates were inoculated by dipping sterile cotton swabs into the suspension of the overnight growth of the organism prepared to a density of McFarland No.0.5 standard; expressed excess liquid from the swabs and inoculated the surface of the agar by spread method. The $1 \mu \mathrm{g}$ oxacillin discs (Oxoid) were aseptically placed on the surface of the inoculated plates and incubated aerobically at $35^{\circ} \mathrm{C}$ for 18 -24hours. The zones of inhibition were measured and compared with CLSI (2010). The isolates that were resistant to oxacillin ( $\leq 10 \mathrm{~mm}$ diameter) were termed methicillin resistant S. aureus (MRSA) (CLSI, 2010).

\section{Data analysis}

Data obtained from the study were analysed using EPI INFO Version 6 for chi-squared test. Comparisons of other parameters were done by simple percentages.

\section{Ethical considerations}

Ethical clearance was approved for the research study by the management of Murtala Mohammed Specialist Hospital, Kano Northwestern Nigeria.

\section{Results}

A total of 2,920 subjects were involved in this study. The age group 31 - 40yrs was observed to have the least infection rate while $>70 y r s$ had the highest. The difference was statistically significant $\left(\chi^{2}\right.$ trend $=4.57 p=0.03$ ) (Table 1$)$. The infection rate by service units among surgical patients shows that the least infection rate was observed in the female surgical ward while the highest was seen at the Male ward D (mostly urogenital patients) (Table 2). When all the wards were compared together there was a significant difference in the infection rate $\left(\chi^{2}=88.4 \mathrm{df}=4\right.$ $\mathrm{p}<0.0001)$. When the ward comprising only females were compared, a statistically significant difference in the infection rate was observed $\left(\chi^{2}=72.58 \mathrm{df}=2 \mathrm{p}<0.0001\right)$. When the wards comprising only male was analyzed, there was also a significant difference observed $\left(\chi^{2}=10.17\right.$; $\mathrm{df}$ $=1 \mathrm{p}<0.001)$.

Table 1: Age, sex distribution and infection rate among surgical wound infected patients in MMSH

\begin{tabular}{llllll}
\hline Age & Male (\%) & Female $(\%)$ & No. of subjects & No. infected & Infection rate(\%) \\
\hline $11-20$ & $68(21.5)$ & $126(22.1)$ & 587 & $194(22.1)$ & 33.0 \\
$21-30$ & $65(20.5)$ & $257(45.7)$ & 1100 & $322(36.7)$ & 29.3 \\
$31-40$ & $63(19.9)$ & $93(16.5)$ & 675 & $156(17.8)$ & 23.1 \\
$41-50$ & $44(13.9)$ & $39(6.9)$ & 257 & $83(9.4)$ & 32.3 \\
$51-60$ & $25(7.9)$ & $31(5.5)$ & 136 & $56(6.4)$ & 41.2 \\
$61-70$ & $30(9.4)$ & - & 85 & $30(3.4)$ & 35.3 \\
$>70$ & $21(6.6)$ & $16(22.8)$ & 80 & $37(4.2)$ & 46.2 \\
Total & 316 & 562 & 2920 & 878 & 30.1 \\
\hline$\chi_{\text {trend }}^{2}=4.57 \mathrm{P}=0.03$ & & &
\end{tabular}


Table 3 shows the frequency of isolates from pure culture from surgical site infection patients in MMSH. E. coli (25.5\%) was the most frequently isolated bacteria followed by S. aureus (20.6\%) and Proteus mirabilis (13.5\%) Bacteroides fragilis (2.0\%) was the most frequently isolated anaerobe followed by Peptococcus spp. (1.4\%). Candida spp. (1.0\%) was also observed. Most common isolates from mixed culture were E. coli+P. mirabilis (18), S. aureus $+P$. vulgaris (10) and Peptostreptococci+S. aureus (6) (Table 4).

Table 2: Infection rate by service units among surgical patients at MMSH

\begin{tabular}{lll}
\hline Service units & Number (\%) of patients & No. (\%) of patients infected \\
\hline Maternity ward & $1161(39.8)$ & $446(38.4)$ \\
Female ward A & $428(14.6)$ & $94(21.9)$ \\
Female surgical ward & $294(10.1)$ & $50(17.0)$ \\
Male surgical ward & $668(22.9)$ & $163(24.4)$ \\
Male ward D & $369(12.6)$ & $125(33.8)$ \\
Total & 2920 & $878(30.1)$ \\
\hline$\chi^{2}=88.4, \mathrm{df}=4 \mathrm{p}<0.0001$ & &
\end{tabular}

Polymicrobial cultures were frequently observed with P. mirabilis occurring 18 times together with E. coli, while S. aureus and P. vulgaris also occurred together for 10 times. Also two cultures were observed to yield three different organisms each. While one of the cultures yielded $P$. mirabilis, E. coli and S. aureus, the other yielded P. vulgaris, E. coli and S. aureus. The total number of isolates reported in this study was 919. While 603 (65.6\%) were Gram negative, 316(34.4\%) were Gram positive bacteria. A ratio of 2.9:1 was observed.

Table 3: Species and frequency of isolates in pure culture from surgical wound infection in patients

\begin{tabular}{lll}
\hline Isolate & No. of isolates & Total isolates \\
\hline E. coli & 197 & 197 \\
P. mirabilis & 95 & 95 \\
P. vulgaris & 33 & 33 \\
Coagulase negative Staphylococcus & 23 & 23 \\
E. faecalis & 32 & 32 \\
S. aureus & 164 & 164 \\
Streptococcus spp. & 28 & 28 \\
P. aeruginosa & 68 & 68 \\
C. freundii & 16 & 16 \\
Candida spp. & 9 & 9 \\
K. pneumonia & 45 & 45 \\
M. morgana & 3 & 3 \\
B. fragilis & 14 & 14 \\
Clostridium spp & 2 & 2 \\
Peptostreptococcus spp & 12 & 12 \\
Peptococcus spp & 13 & 13 \\
Fusobacterium spp & 4 & 4 \\
Serracia marcescens & 2 & 2 \\
Enterobacter spp. & 3 & 3 \\
Total & 840 & 919 \\
\hline
\end{tabular}

The traditional wound classification showed the infection rate a follows; clean wound $8.4 \%$, clean contaminated $38.6 \%$ contaminated $45.0 \%$ and dirty infected $70.0 \%$. S. aureus and Coagulase negative Staphylococcus (COANS) were commonly isolated in clean wounds while bacteria of the enterobacteriacea and anaerobes were the isolates from the other types of wounds. 
Out of a total of 193 isolates of S. aureus observed 69(35.7\%) were methicillin resistant when tested. The cephalosporins, ceftazidime and ceftriaxone including the fluoroquinolone, ofloxacin showed above $70 \%$ sensitivity to most Gram negative bacteria while cotrimoxazole and ampicillin were ineffective (Table 5).

Table 4: Species and frequency of isolates in mixed culture from surgical wound infection in patients

\begin{tabular}{lll}
\hline Isolate & No. of isolates & Total isolates \\
\hline E. coli + P. mirabilis & 18 & 36 \\
E. coli + Streptococcus spp. & 5 & 10 \\
E. coli + Proteus vulgaris & 4 & 8 \\
E. coli + Staphylococcus aureus & 2 & 4 \\
E. coli + Enterococci spp & 3 & 6 \\
P. aeruginosa + P. mirabilis & 2 & 4 \\
K. pneuminiae + P. vulgaris & 2 & 4 \\
P. mirabilis + S. aureus & 7 & 14 \\
P. aeruginosa + E. Coli & 2 & 4 \\
E. faecalis + K. Pneumonia & 1 & 2 \\
S. aureus + P. vulgaris & 10 & 20 \\
S. aureus + P. aeruginosa & 3 & 6 \\
Candida spp + Escherichia coli & 2 & 4 \\
P. mirabilis+E. coli+S. aureus & 1 & 3 \\
P. vulgaris+Enterobacter+E. Coli & 1 & 3 \\
Bacteriodes+E. Coli & 4 & 8 \\
Peptostreptococci+S. aureus & 6 & 12 \\
Fusobacterium+P. mirabilis & 4 & 8 \\
\hline
\end{tabular}


Table 5: Antibiotic susceptibility pattern of isolates from surgical wound infection in patients from MMSH

\begin{tabular}{|c|c|c|c|c|c|c|c|c|c|c|c|}
\hline \multirow[t]{2}{*}{ Pathogens } & \multirow{2}{*}{$\begin{array}{l}\text { Total no. of } \\
\text { isolates }\end{array}$} & \multicolumn{10}{|c|}{ No. (\%) of isolates sensitive to } \\
\hline & & CAZ & OFX & AMC & CXC & $\mathrm{CN}$ & COT & CRO & ERY & CIP & AMP \\
\hline E. coli & 239 & $192(80.3 \%)$ & $180(75.3 \%)$ & $155(64.8 \%)$ & ND & $144(60.3 \%)$ & $6(2.5 \%)$ & $202(84.5 \%)$ & ND & $128(53.5 \%)$ & $18(7.5 \%)$ \\
\hline P. mirabilis & 127 & $93(73.2 \%)$ & $99(77.9 \%)$ & $72(56.6 \%)$ & ND & $81(63.7 \%)$ & $0(0 \%)$ & $80(62.9 \%)$ & ND & $65(51.2 \%)$ & $10(7.8 \%)$ \\
\hline P. vulgaris & 50 & $36(72.0 \%)$ & $35(60.0 \%)$ & $28(56.0 \%)$ & ND & $32(64.0 \%)$ & $0(0 \%)$ & $38(76.0 \%)$ & ND & $26(52.0 \%)$ & $8(16.0 \%)$ \\
\hline CN-Staphylococcus & 23 & $13(56.0 \%)$ & $15(65.2 \%)$ & $12(52.1 \%)$ & $18(78.0 \%)$ & $13(56.0 \%)$ & $\mathrm{o}(0 \%)$ & $13(56.0 \%)$ & $18(78.0 \%)$ & $16(69.5 \%)$ & $0(0 \%)$ \\
\hline E. faecalis & 36 & $22(61.1 \%)$ & $21(58.3 \%)$ & $18(50.0 \%)$ & $15(41.6 \%)$ & $15(41.6 \%)$ & $4(11.1 \%)$ & $24(66.6 \%)$ & $18(50.8 \%)$ & $20(55.5 \%)$ & $5(13.8 \%)$ \\
\hline S. aureus & 193 & $156(80.8 \%)$ & $160(82.9 \%)$ & $120(62.1 \%)$ & $164(84.9 \%)$ & $119(61.6 \%)$ & $0(0 \%)$ & $150(77.7 \%)$ & $124(64.5 \%)$ & $120(62.1 \%)$ & $0(0 \%)$ \\
\hline Streptococcus spp. & 33 & $12(36.4 \%)$ & $14(42.4 \%)$ & $12(33.3 \%)$ & $19(57.5 \%)$ & $12(33.3 \%)$ & $2(6.1 \%)$ & $17(51.5 \%)$ & $15(55.5 \%)$ & $12(33.3 \%)$ & $3(9.1 \%)$ \\
\hline$P$. aeruginosa & 75 & $57(76.0 \%)$ & $59(78.6 \%)$ & $1(1.3 \%)$ & ND & $50(66.6 \%)$ & $\mathrm{o}(0 \%)$ & $56(74.7 \%)$ & ND & $25(33.3 \%)$ & $\mathrm{o}(0 \%)$ \\
\hline C. freundii & 16 & $10(62.5 \%)$ & $12(75.0 \%)$ & $8(50.0 \%)$ & ND & $8(50.0 \%)$ & $0(0 \%)$ & $11(68.8 \%)$ & ND & $8(50.0 \%)$ & $2(12.5 \%)$ \\
\hline K. pneumoniea & 48 & $35(72.9 \%)$ & $34(70.8 \%)$ & $25(52.1 \%)$ & ND & $28(58.3 \%)$ & $0(0 \%)$ & $35(72.9 \%)$ & ND & $30(62.5 \%)$ & $4(8.3 \%)$ \\
\hline M. morgana & 3 & $2(66.6 \%)$ & $3(100.0 \%)$ & $1(33.3 \%)$ & ND & $\mathrm{o}(0 \%)$ & $0(0 \%)$ & $3(100 \%)$ & ND & $0(0 \%)$ & $0(0 \%)$ \\
\hline S. marsescenes & 2 & $2(100.0 \%)$ & $1(50 \%)$ & $1(50 \%)$ & ND & $1(50 \%)$ & $\mathrm{o}(0 \%)$ & $1(50.0 \%)$ & ND & $1(50.0 \%)$ & $\mathrm{o}(0 \%)$ \\
\hline Enterobacter spp & 4 & $3(75 \%)$ & $3(75 \%)$ & $2(50.0 \%)$ & ND & $2(50 \%)$ & $0(0 \%)$ & $3(100 \%)$ & ND & $4(100 \%)$ & $0(0 \%)$ \\
\hline
\end{tabular}

CAZ-Ceftazidime, OFX-Ofloxacin, AMC-Amoxicillin/clavulanate, CXC-Cloxacillin, COT-Cotrimoxazole, CRO-Ceftraxone, ERY-Erythromycin, CIP-Ciprofloxacin, AMPAmpicillin, ND-Not done; $\mathrm{CN}=$ coagulase negative 


\section{Discussion}

Once a patient's major defence against infection, the intact skin, is breached by either trauma or surgical knife, a broad avenue is opened to introduction of virulent bacteria (Enhrenkranz \& Meakins, 1992). Multiplicity of factors influence SSI rate in clinical practice. This could result from the patient undergoing surgery, members of the operating room or the operating room environment. The pathogens isolated from infections differ primarily depending on the surgical procedure. In clean surgical procedures, in which the gastrointestinal, gynaecologic and respiratory tracts have not been entered, S. aureus from the exogenous environment or the patient's skin flora is the usual cause of infection (Nichols, 1984). In other categories of surgical procedures, including clean-contaminated, contaminated and dirty, the polymicrobial aerobic and anaerobic flora closely resembling the normal endogenous microflora of the surgically resected organ are the most frequently isolated pathogens (Nichols, 1984). This is in agreement with the observation in this study.

In the present study E. coli was the most frequently isolated bacterium and also reflected in the reports of Jan et al. (2010) and Shah et al. (2010). However, the above observation is at variance with the findings from some other workers who observed Staphylococcus aureus as the most frequently isolated organism in their different centres (Isibor et al., 2008; Abu, 1990; TwumDanso et al., 1992). The recovery of Candida spp. in this study also agrees with the finding of Isibor et al. (2008) in Nigeria.

Polymicrobial cultures were observed in this study where a mixture of two and three different bacterial isolates was obtained in significant proportions. Ganguly et al. (2000) reported a similar observation in their study. The high ratio of Gram negative bacteria observed over Gram positive bacteria in this study agrees with the report of Oni et al. (2001). Methicillin resistant S. aureus had a prevalence rate of $35.7 \%$ which compares favorably with the observations of Taiwo et al. (2005) though lower than the report from studies in Jos, Nigeria (Ikeh, 2003).

The overall infection rate of $30.1 \%$ observed in this study is in agreement with the findings of Kamat et al. (2008) but higher than in more developed countries (Dellinger \& Enhrenkranz, 1998). A report from Tehran (Arabshahi \& Koohpayezade, 2006) reported an infection rate of 8.4\% while that from Tanzania (Eriksen et al., 2003) reported an infection rate of $19.4 \%$. The hospital environment supports the acquisition of resistance to antibiotic agents by pathogens, complicating the treatment of infections due to drug resistant pathogens (Emori \& Gaynes, 1993). Commonly used antimicrobial agents such as cotrimoxazole and ampicillin were found to be ineffective in the in vitro sensitivity. Cephalosporins such as ceftriaxone and ceftazidime including the fluoroquinolone ofloxacin gave encouraging results. These observations are in agreement with the reports of Mahesh et al. (2010).

Studies on surgical site infection should be carried out regularly in each locality to establish any changes in the pattern of antibiotic resistance as well as spectrum of prevalent pathogens. Baseline data obtained from such studies will be of clinical relevance in guiding antibiotic prescription policies for prophylaxis and therapeutic purposes. This will be very helpful in reducing $\mathrm{SSI}$ in this environment.

\section{References}

Abu, H.Y. (1990) Postoperative surgical wound infection. Medical Journal of Malaysia 45, 293-297.

Angue, J.R. \& Olila, D. (1999) Drug sensitivity patterns of bacterial isolated from septic postoperative study of 3280 surgical wounds. Indian Journal of Medical Microbiology 17, 129-132.

Anvikar, A.R., Desmukh, A.B., Karyakrte, R.P., Damble, A.S., Patvardan, N.S., Malik, A.K. et al. (1999) A one year prospective study of 3280 surgical wounds. Indian Journal of Medical Microbiology 17, 129-132. 
Baron, E.J.O., Peterson, L.R. \& Finegold, S.M. (1994) Processing clinical specimens for anerobic bacteria: Isolation and identification procedures, In: Bailey and Scott's Diagnostic Microbiology 9th Edition, Mosby - Year book Inc. Missouri, pp. 480-484.

Bauer, A.W., Kirby, W.M.M., Sherris, J.C. \& Turck, M. (1996) Antibiotic susceptibility testing by a standardized single disc method. American Journal of Clinical Pathology 45, 493-496.

Cheesbrough, M. (1993) Medical laboratory manual for tropical countries. Microbiology Vol II, 200-287pp.

CLSI (2012) Performance standards for antimicrobial susceptibility testing; Twenty-Seond Informational Supplement. Clinical and Laboratory standards institute. M100-S22 Vol 32 No. 3

Dellinger, E.P. \& Enhrenkranz, N.J. (1998) Surgical Infections, in hospital infections, $4^{\text {th }}$ edn. (Ed: J.V. Bennett \& P.S. Brachmann). Lippincott-Raven Publishers, Philadelphia.

Ejikeme, N. (2001) Bacteriology of postoperative wound infections in the surgical ward of a federal medical centre in Nigeria. Journal of Medical Investigation and Practice 3, 6064.

Emori, T.G. \& Gaynes, R.P. (1993). An overview of nosocomial infection including the role of Microbiology Laboratory. Clinical Microbiology Reviews 6, 428-442.

Enhrenkranz, N.J. \& Meakins, N. (1992) Surgical Infections In: Hospital infections (Ed) by JV Beneth and PS Brachman (eds), pp 685-710.

Eriksen, H.M., Chugulu, S., Kondo, S. \& Lingaas, E. (2003 Surgical site infections at Kilimanjaro Christian Medical Centre. Journal of Hospital Infection 55,14-20.

Ganguly, P.S., Khan, Y. \& Malik, A. (2000) Nosocomial infections and hospital procedures. Indian Journal of Community Medicine 25, 990-1014.

Ikeh, E.I. (2003) Methicillin-resistant Staphylococcus aureus (MRSA) at Jos University Teaching Hospital. African Journal of Clinical and Experimental Microbiology 4, 52-55.

Isibor, J.O., Osemi, A., Eyaufe, A., Rachael, O. \& Turay, A. (2008) Incidence of aerobic bacteria and Candida albicans in Postoperative wound infections. African Journal of Microbiology Research 2, 288-291.

Jan, W.A., Khan, M.S., Jehanzeb, M. \& Muazzam (2010) Surgical site infection and pattern of antibiotic use in a tertiary care hospital in Peshwar. Journal of Ayub Medical College Abbottabad 22, 141-145.

Kamat, U.S., Fereirra, A.M.A., Kulkarni, M.S. \& Motghare, D.D. (2008) A prospective study of surgical site infections in a teaching hospital in Goa. Indian Journal of Surgery 70, 120124.

Kumar, A.P., Mithelesh, A., Ashkok, B. \& Halim, T. (1985) Epidemiology of Pseudomonas aeruginosa; post-operative wound sepsis. Indian Journal of Pathology and Microbiology 28, 137-146.

Linani, S.P., Jangali, N., Chowdhary, A. \& Daver, G.B. (2005) Surgical site infection in clean and clean contaminated cases. Indian Journal of Medical Microbiology 23, 249-252.

Mahesh, C.B., Shivakumar, S., Suresh, B.S., Chidanamel, S.P. \& Vishwanath, Y. (2010) A prospective study of surgical site infections in a teaching hospital. Journal of Clinical and Diagnostic Research 4, 3114-3119.

Nichols, R.L. (1984) Prevention of infection in high risk gastrointestinal surgery. American Journal of Medicine 76, 111-119.

Nichols, R.L. (2001) Preventing Surgical site infection: A surgeon's perspective. Emerging Infectious Diseases 7, 220-224.

Oni, A.A., Eweta, A.F., Gbaja, A.T., Kolade, A.F., Matiu, W.B., Adeyemo, D.A. \& Bakare, A.R. (2006) Nosocomial infections: surgical site infection in UCH Ibadan, Nigeria. Nigerian Journal of Surgical Research 8, 19-23.

Shah, F., Gandhi, M., Mehta, V., Udani, D., Mundra, M. \& Swadia, N. (2010) Nosocomial infections in Surgical Wards. Internet Journal of Surgery 24(1). 
Taiwo, S.S., Bamidele, M., Omonigbehin, E.A., Akinsinde, K.A., Smith, S.I., Onile, B.A. \& Olowe, A.O. (2005) Molecular epidemiology of Methcillin resistant Staphylococcus aureus in Ilorin, Nigeria. West African Journal of Medicine 24(2), 100-106.

Twum-Danso, K., Grant, C., Al-Suleiman, S.A., Abdel-Khader, S., al-Awami, M.S., al-Breiki, H. Taha, S., Ashoor, A.A. \& Wosornu, L. (1992). Microbiology of postoperative wound infections: a prospective study of 1770 wounds. Journal of Hospital Infection 21(1):2939.

Waqar, A.J., Sajjad, M.K. \& Muhammed, J., Muzzam (2010). Surgical site infection and pattern of antibiotic use in a tertiary care hospital in Peshwar. Journal of Ayub Medical College Abbottabad 22, 141-145. 